\title{
MSCs derived from Placenta-choriodecidual Membrane Attenuate Lipopolysaccharide induced-Acute Lung Injury through Mediating the Polarization of Pro-inflammatory Macrophages
}

\author{
Chia-Ling $\mathrm{Wu}^{1}$, Meng-Shiue $\mathrm{Wu}^{1,2}$, Ching-Liang $\mathrm{Chu}^{3}$, Thai-Yen Ling ${ }^{1}$ \\ ${ }^{\prime}$ Department and Graduate Institute of Pharmacology, College of Medicine, National Taiwan University, Taiwan, \\ ${ }^{2}$ Department of Biochemistry and Molecular Cell Biology, School of Medicine, College of Medicine, Taipei Medical \\ University, Taiwan, ${ }^{3}$ Graduate Institute of Immunology, College of Medicine, National Taiwan University, Taiwan
}

\section{Background}

Acute lung injury (ALI) is considered to be leading cause of death in clinical care. Although many studies have made intensive efforts to reveal optimal treatments for the disease, the therapeutic options are still limited and the mortality rate remains high at 40-60\%. Instead, supportive care is utilized with agents include corticosteroids, neuromuscular blocking agents, nitric oxide, surfactants, and $\beta_{2}$-adrenergic agonists. These supportive cares could contribute to decrease mortality of ALI, however, the regeneration for the damaged pulmonary tissue and end-staged fibrosis remain incurable. The lacks of effective treatment make it essential to develop new therapeutic medicines. Mesenchymal stem cells (MSCs) have been reported to have the capacity of immunomodulation and tissue regeneration in vitro and in vivo, therefore MSCs could to be a promising regenerative medicine for ALI.

\section{Methods}

We applied MSCs derived from placenta-choriodecidual membranes, named pcMSCs, which established by a serum-free culture condition, to investigate the therapeutic efficacy of pcMSCs in in vitro and in vivo. We first used LPS to stimulate bone marrow-derived monocyte (BMDMs) and cocultured with pcMSCs as an in vitro model. In ALI animal model, $400 \mathrm{ug}$ of LPS was used to treat BALB/c mice by intratracheal; 24 hours after LPS treatment, $5 \times 10^{5}$ pcMSCs were injected by tail-veined injection. In the animal model, Bronchial-alveolar lavage were collected to evaluate the M1/M2 shifting in macrophages by flow cytometry after pcMSCs cell therapy. Meanwhile, histological sections and ventilation measurements were also performed to evaluate therapeutic effects for the cells.

\section{Results}

Our results suggested that pcMSCs could uppress maturation and differentiation of LPS-treated BMDMs in vitro. The pro-inflammatory cytokine secreted by BMDMs were also down-regulated by pcMSCs, while COX-2 of pcMSCs were activated. The flow cytometry analysis for BMDMs indicated that pcMSCs were able to promote LPS-induced CD86 ${ }^{+}-$ M1-liked macrophages shifting to a CD206-M2-liked phenotype. In animal model, pcMSCs could also reduce leukocyte infiltration, tissue damage and mortality rate of BALB/c mice after LPS induced.

\section{Conclusions}

These results showed that pcMSCs could serve as a promising cell therapy source for ALI animal model and the effects may come from contact-independent soluble factors. 